\title{
Arreglándoselas con la tradición: Rorty y los usos del legado filosófico
}

\section{Coping with tradition: Rorty and the uses of the philosophical legacy}

\author{
JOSÉ A. MARÍN-CASANOVA \\ Universidad de Sevilla
}

Recibido: 25-02-2010 Aprobado definitivamente: 10-05-2010

\begin{abstract}
RESUMEN
El objetivo de este trabajo es mostrar que la relación de Rorty con la tradición filosófica presenta muchos más matices de los que su reputación podría hacer creer. Se confrontan dos hechos: de un lado, el propósito rortiano de superar la tradición; de otro, la necesidad de la tradición para mantener la conversación, interés principal de la filosofía para Rorty. Por tanto, la relación de Rorty con la tradición es ambigua. Ahora bien, esa ambigüedad puede verse como no contradictoria: Rorty no pretendió tanto ofrecer una argumentación alternativa a la de la tradición cuanto una alternativa a la argumentación de la tradición. Así, más que romper con la tradición, Rorty, a su modo, le da una oportunidad.
\end{abstract}

PALABRAS CLAVE

RORTY, TRADICIÓN, FINAL DE LA FILOSOFÍA, (NEO)PRAGMATISMO, RETÓRICA

\section{ABSTRACT}

The aim of this paper is to show that Rorty's relationship with philosophical tradition has more nuances of meaning than his reputation might suggest. Two opposite facts are considered: on the one hand, Rorty's intention to overcome tradition; on the other hand, the fact that there cannot be conversation, the main interest of philosophy for Rorty, without tradition. Rorty's relationship to the philosophical tradition is, thus, ambiguous. Nevertheless, this ambiguity can be seen as not inconsistent. One reason might explain this fact: rather than an alternative argumentation to the traditional one, Rorty offered an alternative to the traditional argumentation. Therefore it makes more sense to read Rorty as giving the tradition a break rather than breaking with the tradition.

\section{KEYWORDS}

RORTY, TRADITION, END OF PHILOSOPHY, (NEO)PRAGMATISM, RHETORIC

C Contrastes. Revista Internacional de Filosofía, vol. XVI (2011), pp. 271-289. ISSN: 1136-4076 Departamento de Filosofía, Universidad de Málaga, Facultad de Filosofía y Letras

Campus de Teatinos, E-29071 Málaga (España) 
RORTY NUNCA DEDICÓ NINGÚN estudio monográfico a la tradición. La palabra «tradición», si bien salpica muchas de sus páginas, sólo aparece en dos títulos suyos. ${ }^{1}$ Sin embargo, el proyecto intelectual rortiano - sedicentemente utópico ${ }^{2}$ - hay que entenderlo, según la intentio auctoris, como persecución del logro de una «cultura postFilosófica» ( $c f$. CP xxxvii-xliv), y con independencia de lo que la caracterización explícita de esa cultura pueda decirnos, el empleo del prefijo «post» lleva aparejado implícitamente ya el dejar a un lado la tradición filosófica, disponerse a ir más allá de la Filosofía poniéndose al margen de su tradición. La consecución de este objetivo cultural lleva consigo, por definición, un rechazo cierto de la tradición filosófica, toda una proclamación de su inactualidad. De hecho, en la obra rortiana se dan expresiones explícitas alentadoras del olvido de «las viejas malas cuestiones» o de «abandonar nuestro pasado intelectual», si no de «minar la tradición» como algo obligatorio: «debemos abandonar el proyecto filosófico tradicional» (RFM, 31). Prima facie, la entera obra rortiana responde efectivamente a este aliento y ello se plasma en su planteamiento «antifundacionalista» (en lo ontológico), «antirrepresentacionalista» (en lo gnoseológico) y «etnocentrista» (en lo axiológico), los tres pivotes del pensamiento rortiano y metonímicamente de toda la «cultura postFilosófica».

1 Este trabajo ha sido realizado dentro del Proyecto de Investigación (I+D+i) FFI200907709 del Ministerio de Ciencia e Innovación.

«Overcoming the Tradition: Heidegger and Dewey» (CP, 37-59), y «Derrida and the Philosophical Tradition» (TP, 327-50). También aparece el término «tradición» en el primer epígrafe de «Philosophy in America Today» (CP, 211-30), donde se pronuncia sobre «Analytic Philosophy and the Tradition», para distinguir entre estas «dos tradiciones institucionalizadas» ( $c f$. EHO, 22): el empirismo lógico es antes consecuencia de una tradición, que la crítica (lógicamente) «neutra», que creía ser, de la tradición ( $c f$. CP, 75). Por otro lado, una muestra del supuesto «desinterés» de Rorty por la tradición es su falta de respuesta explícita a la pregunta «¿Qué aporta el conocimiento de la tradición filosófica [...]?», limitándose en la contestación, sin mencionar la palabra «tradición», a lamentar el daño que en los países anglosajones ha supuesto el descuido de la historia de la filosofía ( $c f$. PGT, 75-6); y ante la cuestión «¿hacerse cargo de los problemas de la tradición filosófica no tiene valor especial?» sólo responde: «Bueno, conseguir que los estudiantes se devanen los sesos con la oposición entre libertad y determinismo o con la existencia del mundo material sí que tiene valor, aunque en ello entra en juego algo de astucia. Así se consigue que los estudiantes lean libros que deberían leer [...]. Si no [...] se corre el riesgo de que ellos mismos los escriban»(PGT, 78-9), aunque enseguida reconoce que leer el canon nos hace más prudentes y capaces de medir nuestra contingencia.

2 Por cierto, «en este futuro utópico las tradiciones culturales ya no influirán en las decisiones políticas. En política habrá una sola tradición: la de la vigilancia constante sobre los intentos previsibles del rico y fuerte de aprovecharse del pobre y débil poniéndolo en desventaja» (PF, 204). 
En efecto, al trazar el perfil de esos pivotes Rorty está dibujando per negationem el de la cultura filosófica, es decir, la «tradición», que ha de quedar necesariamente atrás. Esa negación de la tradición unas veces es latente y otras, las más, es patente, aunque cambie el grosor del trazo. Cuando el trazo es grueso Rorty no distingue entre unas tradiciones y otras, y todas entran en el saco común de la tradición que denomina ora «filosófica», ora «metafísica», ora «esencialista», «objetivista», ora «occidental». Cuando el trazo se afina Rorty suele distinguir, sobre todo, entre la «tradición platónica» (o en alguna ocasión la «tradición parmenídea») para referirse a la Filosofía en general, y la «tradición kantiana» (o en alguna ocasión la «tradición cartesiana» o «postrenacentista»o «racionalista») para referirse, por antonomasia, a la Filosofía moderna. No se trata de elaborar un catálogo analizando cada referencia rortiana a esas tradiciones. Aquí vamos a permitirnos, por el contrario, tomarnos la misma licencia con Rorty que éste se toma con la tradición: con él y como él no vamos a utilizar el trazo fino, sino que nos limitaremos pragmáticamente a señalar el uso negativo que hace Rorty de la tradición para en su desmarque presentar su propuesta positiva, aunque adelantamos que es un autor más destruens que construens, ${ }^{3}$ hasta el punto de preguntarnos si no podemos sospechar de él lo que él mismo interpreta que venía a afirmar Dewey: «Me limito a quitar de en medio los árboles muertos de la tradición filosófica» (CP, 73). Un lector poco propicio a Rorty incluso podría someter a retorsión la acusación que, sin menoscabo de su profesada admiración por él (cf. RFM, 32), hace del viejo pragmatista cuando pone de relieve «un error que se reitera en la obra de Dewey: su hábito de anunciar un programa audaz, nuevo y positivo, cuando todo lo que ofrece, y todo lo que tiene que ofrecer es una crítica de la tradición» (CP, 78). Aunque, a fuer de sinceros, esa eventual retorsión no sería del todo justa, ya que Rorty no se afana tanto en proclamar un programa, por mucho que su utopía liberal, ironista y solidaria lo pueda parecer, cuanto en ofrecer una peculiar crítica a la tradición, una crítica que a la vez que la quiere superar la parasita.

Que se trata de una crítica parasitaria no es una exageración nuestra (cf. PF, 199). Rorty es consciente de necesitar la tradición, de que sin ella se quedaría sin tema y perdería su ironía. Rorty tiene en la tradición su contrapunto dialéctico, en cuya ausencia no tendría nada que decir. La actitud irónica del «modernismo», que él hace suya, en conformidad con el carácter reactivo por él atribuido al pragmatismo ( $c f$. PMN, 369, 378; RFM, 32), «sería imposible sin el concurso de una animada tradición filosófica que mantiene viva la imagen imagen de la mente o del lenguaje. Sin la lucha predestinada al fracaso de los filósofos por inventar una forma de representar que nos obligue a la verdad

3 En Rorty destaca más lo que rechaza que lo que propone ( $c f$. Abrams 2002). 
mientras nos libere del error, por hallar imágenes donde sólo hay juegos, no habría nada respecto de lo cual ser irónico. En una cultura donde no hubiese diferencia alguna entre ciencia y poesía, tampoco habría poesía sobre la poesía, ni escritura que fuese glorificación de la escritura misma. Es precisamente este contraste el que nuestros filósofos parmenídeos mantienen vivo para nosotros» $(\mathrm{CP}, 136)$. Es precisamente esa cultura no dualista la «cultura postFilosófica».

Por eso, sin embargo, sus tres pivotes se definen a redropelo de la bulliciosa tradición. En primer lugar, el antifundacionalismo, que rechaza la madre de toda ontología, la dicotomía realidad/apariencia. Y no, desde luego, para hacer de la apariencia realidad, sino para salirse de cualquier jerarquía que divida lo que hay en un sector realmente real y otro aparentemente real. Rorty prefiere reemplazar esa dualidad para poner en su lugar otras como la contraposición entre pasado y futuro ( $c f$. PSH, 24) o entre lo útil y lo inútil. ${ }^{4}$ De hecho, se vale de la distinción entre lo más y lo menos útil para esquivar la cuestión ontológica. El antiesencialismo rortiano nunca afirma que no haya fundamento, simplemente no considera útil su cuestión. De modo que no se trata de que no exista aquello de lo que la Metafísica se ha ocupado tradicionalmente, mientras que sí existiría aquello de lo que se ocupa la tradición pragmatista en la que se autoinserta Rorty. El asunto no es el de la existencia del fundamento sino el de su utilidad. Fundamento y utilidad están en planos distintos: no es que lo real sea lo útil y lo aparente lo inútil, sino que la bina fundamental entre lo real y lo aparente, que tan útil le fue a la tradición, simplemente ya no es útil (cf. RFM, 37). Con lo que «Rorty sacrifica tradición filosófica por eficiencia» ${ }^{5}$ y el deseo de realidad tradicional por el deseo de futuro.

4 En el «Prólogo del autor a la presente edición» española de CP leemos: «he llegado al convencimiento de que la doctrina central del pragmatismo representa la propuesta de reemplazar la distinción entre apariencia y realidad [...] por la distinción entre descripciones más útiles y descripciones menos útiles de las cosas» (R. Rorty, Consecuencias del pragmatismo, tr. José M. Esteban. Madrid: Tecnos, 1996, p. 13 [ $c f$. RFM, 37; PSH, 27, 48]). Esta «doctrina central» tiene a su base la estrategia retórica de la «redescripción» como «alternativa» a la actitud teorética tradicional. Rorty no tiene una teoría de la realidad ni del conocimiento ni de la acción; antes al contrario, se dedica a redescribir esos expedientes con la convicción «ironista» de la contingencia de nuestro lenguaje y, por tanto, de que lo único que tenemos son descripciones alternativas sin alternativas no descriptivas, y nunca la única descripción correcta, pues el mundo no nos proporciona criterio alguno para decidir entre las alternativas ( $c f$. CIS, 3-22; Calder 2003).

5 Ocampo 2004. 
Y es que quizá se pueda leer la empresa intelectual rortiana, en general, y contra la tradición, en particular, como descarte de la idea platónico-kantiana de fundamentación, en la cual Rorty ve un rasgo distintivo de la tradición filosófica, es decir, como «el intento de liberar a la humanidad de la 'mentira más duradera' que Nietzsche desenmascara, la idea de que fuera de los experimentos fortuitos y peligrosos que hacemos yace algo (Dios, Ciencia, Conocimiento, Racionalidad, o Verdad) que, sólo con seguir los ritos correctos, vendrá a salvarnos» (CP, 208). Se trata, entonces, de librarnos de la tentación tradicional de fundamentar nuestra cultura sobre bases filosóficas, tentación en que cayeron la «tradición platónica» y la «kantiana», caída que las unifica. En efecto, el platónico «no quiere estar meramente sometido a las disciplinas del presente, sino a la naturaleza ahistórica e inhumana de la realidad en sí. Este impulso cobra dos formas: la inicial estrategia platónica de postular nuevos objetos para corresponder con proposiciones atesoradas y la estrategia kantiana para hallar principios que definan la esencia del conocimiento, de la representación, de la moralidad o de la racionalidad. Pero esta diferencia carece de importancia comparada con el común afán de escapar del vocabulario y las prácticas de nuestro tiempo y descubrir algo ahistórico y necesario a lo que aferrarnos [...]. Este afán [...] es definitorio de la tradición filosófica occidental, y de la cultura para la cual habla esta tradición» $(\mathrm{CP}, 165)$.

En segundo lugar, el antirrepresentacionalismo, que rechaza la madre de toda la gnoseología, la dicotomía verdad/opinión. Y no, desde luego, para hacer de la opinión verdad, sino para salirse de cualquier jerarquía que divida lo que conocemos en un sector realmente verdadero y otro aparentemente verdadero. La posición propia de Rorty en el ámbito epistemológico parte precisamente del rechazo de un conocimiento mimético. Si pragmáticamente se prescinde de la idea de una realidad realmente real, no cabe entonces plantearse el saber como adecuación: «la noción tradicional de que «a verdad es correspondencia con la realidad> es una metáfora que no se puede hacer efectiva y está desgastada» (ORT, 79). Perdida toda importancia de plantearse la cuestión del Ser, perdida toda importancia de plantarse la cuestión de la Verdad; al menos, de la verdad en mayúsculo singular. Rorty dice siempre no tener ninguna teoría de la verdad. La única acepción de «verdad» con que él trabaja es la de verdad como propiedad de los enunciados, una verdad adjetiva; en ningún caso, como propiedad o atributo del ser o de la realidad, una verdad substantiva. ${ }^{6}$ Carece de utilidad para él lo que es presupuesto implícito de la acepción rechazada, a

6 «Rorty, consistentemente con su anti-representacionalismo, es un minimalista acerca de la verdad» (Nielsen 1999, p. 506): «Cuando hipostasiamos el adjetivo 〈verdadero〉 como 〈Verdad〉 y nos preguntamos por nuestra relación con ella, resulta que no tenemos nada en absoluto que decir» (TP, 4 [cf. TPP, 23]). 
saber, que hay enunciados que no están en contacto con la realidad. Para Rorty, dada su apuesta por un conocimiento productivo en lugar de reproductivo, por una concepción instrumental $l^{7}$ del lenguaje, no hay manera de no estar en la realidad. ${ }^{8} \mathrm{Y}$ es que plantearse la cuestión del «contacto» de la mente con la realidad presupone la posibilidad del no contacto, lo cual presupone, a su vez, la separación previa de la mente y la realidad, es decir, la disyunción polar entre la mente o espacio interior, lo espiritual, y lo otro de la mente o espacio exterior, lo corpóreo.

Rorty prescinde así de «la imagen que mantiene cautiva a la filosofía tradicional», «la mente como un gran espejo» (PMN, 12) de la Naturaleza (u «ojo inmaterial» [LT, 39, n. 75]), y con ella del sentido fuerte tradicional de las dicotomías theoríalphrónesis (o poíesis), epistémeldoxa (o pístis) y transcendental/empírico. Semejantes distinciones son útiles en la vida cotidiana, dentro de un vocabulario común o «normal», pero no entre vocabularios alternativos, para decidir entre ellos: «La gran falacia de la tradición [...] es pensar que las metáforas de la visión [...] aplicables a afirmaciones minúsculas y rutinarias también pueden aplicarse a afirmaciones mayúsculas y discutibles. Este error básico engendra la idea de que donde no hay objetos a los que corresponder no hay esperanza para la racionalidad sino sólo gusto, pasión y voluntad. Cuando el pragmatista ataca la noción de verdad como precisión de representación, está atacando también las distinciones tradicionales entre razón y deseo, razón y apetito, razón y voluntad. Y es que ninguna de estas distinciones tiene sentido a menos que se conciba la razón tomando como modelo la visión» $(\mathrm{CP}, 164) .{ }^{9}$

7 «La tradición filosófica ha ansiado encontrar una forma de aproximarse a la total pasividad del folio en blanco. [...] Las metáforas que, según el pragmatista, ponemos en lugar de toda esa jerigonza masoquista de carácter duro y directo son las de la conducta lingüística como uso de herramientas [...]. El pragmatista exalta así la espontaneidad» (ORT, 81).

8 Hay que «tratar los vocabularios como herramientas en lugar de como espejos» (EHO, 158): «Ver el empleo de palabras como el uso de herramientas [...] equivale a rechazar la pregunta sobre si la mente humana está en contacto con la realidad» (RFM, 38).

9 Esta cita merece un doble comentario. Primero, con los años la expresión «gran falacia» («error típico de la filosofía tradicional» en EHO, 59) habría que entrecomillarla, como forma de hablar (exagerada), no como error lógico; de hecho, el propio Rorty entona una palinodia sobre el uso de estas expresiones, tildadas por Putnam de «desdén carnapiano», ya en 1993 ( $c f$. TP, 45). Segundo, un Leitmotiv rortiano no siempre explícito y presente en esta cita es, contra la tradición, disociar racionalidad de verdad, así como disociar el amor a la sabiduría propio de la tradición del otro elemento tradicional característico, la argumentación (lógica). Esta técnica retórica de la disociación corre paralela y en sentido inverso de la principal técnica rortiana consistente en «borrar las distinciones» para así neutralizar el dualismo tradicional, constituyendo juntas el par de fuerzas que hace rotar esta peonza filosófica. Sobre el calado de superar el dualismo basten dos referencias: «La filosofía antiesencialista 
En tercer lugar, el etnocentrismo ${ }^{10}$ que rechaza la madre de toda la axiología, la dicotomía norma/hecho. Y no, desde luego, para hacer del hecho norma, sino para salirse de cualquier jerarquía que divida lo que hacemos en un sector universalmente «valioso» y otro particularmente «fáctico», puesto que «no hay diferencia epistemológica entre la verdad de lo que debe ser y la verdad de lo que es, ni ninguna diferencia metafísica entre hechos y valores, ni diferencia metodológica entre moralidad y ciencia» $(\mathrm{CP}, 163)$. Rorty desembaraza a la reflexión moral de la tradicional pretensión de una normativa universal, pretensión ligada a una fundamentación objetiva de los principios morales, universales, inmutables y necesarios, sobre los cuales levantar un Bien gemelo de la Verdad y la Realidad. ${ }^{11}$ Frente al Yo transcendental de la tradición, central, ahistórico y trancultural, esencia humana en la que los yoes empíricos reconocerían su identidad, Rorty defiende la contingencia del yo (cf. CIS, 23-43), ${ }^{12}$ miembro de una comunidad también contingente (cf. CIS, 4469), nuestra y no de la naturaleza, formada y no descubierta, un yo reticular, histórico y aculturado, que carece, en consecuencia, del «confort metafísico» de la tradición occidental. El etnocentrismo no significa que por virtud (o verdad) haya que entender lo que una comunidad acepta como tales, pero sí reconocer que todo lo que sobre ella se puede explicar comporta retrotraerse

espera el día en que todos los pseudoproblemas creados por la tradición esencialista [...] se disuelvan. Piensa que todos estos dualismos tradicionales se derrumban [...] tan pronto como se derrumba la distinción entre esencia y accidente» (ORT, 99); y la menos «carnapiana»: «Si tuviéramos que buscar un modo instantáneo de describirnos a nosotros mismos, acaso lo mejor sería llamarnos a nosotros mismos antidualistas» (RFM, 34 [cf. PSH, 5- 7, 12, 27, 30-1, 35-6, 48]).

10 «Preferiríamos morir a ser etnocéntricos, pero el etnocentrismo es justo la convicción de que preferiríamos morir antes que compartir determinadas creencias» (ORT, 203). Si no poder salir de nuestra piel parece «chocante», es porque «somos los herederos de esta tradición objetivista, que se centra en torno a la presuposición de que debemos salirnos fuera de nuestra comunidad tanto tiempo como para examinarla desde algo que va más allá de ella» (ORT, 22), «es porque la tradición filosófica nos ha acostumbrado a la idea de que quienquiera que esté dispuesto a atender a razones [...] puede ser persuadido a reconocer la verdad» (ORT, 88). Ahora bien, Rorty clama por que no se confunda su etnocentrismo con un relativismo según el cual cada comunidad o cada tradición sería tan buena como cualquier otra, lo que no dejaría de presuponer una perspectiva divina (la de un dios indiferente a los léxicos humanos); por el contrario, hace suyas estas palabras de Putnam: «sólo podemos esperar producir una concepción más racional de la racionalidad o una mejor concepción de la moralidad si operamos desde dentro de nuestra tradición» (cf. ORT, 202).

11 «[P]ienso que la retórica que nosotros los occidentales usamos al tratar de hacer que todo el mundo sea más como nosotros mejoraría si fuéramos más francamente etnocéntricos y menos profesamente universalistas» (JLL, 56-7).

12 La concepción descentrada e irónica del sí mismo debe mucho a Freud, confrontado con la tradición filosófica en EHO, 143-63. 
al aspecto concreto de la cultura en que ese término surgió y se desarrolló, a saber, Europa, la cultura occidental.

Es este etnocentrismo post-Filosófico, en que se condensan prácticamente el antifundacionalismo y el antirrepresentacionalismo, donde, abandonados a sí mismos, «hombres y mujeres se sienten solos, meramente finitos, sin vínculos con el Más Allá» (CP, xlii-xliii), libres de toda constricción fundamental, al margen de toda necesidad metafísica, el que, a nuestro juicio, nos proporciona la llave maestra de la «ambigua» relación de Rorty con la tradición, quien a la vez que la abandona aspira a una «nueva tradición intelectual» (CP, xxx). El etnocentrismo nos orienta «sobre cuánto margen de maniobra queda incluso después de que uno llega al convencimiento de que la tradición filosófica ha agotado todas sus posibilidades» (CP, 42), y colma ese espacio sosteniendo que «la mejor esperanza para la filosofía es no practicar Filosofía» (CP, xv), pues paradójicamente «uno puede ser un filósofo precisamente siendo anti-Filosófico, [...] la mejor manera de cohesionar las cosas es [...] abandonar [...] las presuposiciones de la Filosofía» (CP, xvii).

\section{III}

En efecto, en esta «aproximación pragmática a la tradición filosófica» (TP, 332, n. 7) de lo que se trata ya no es de, preservando la «pureza de la Filosofía», «hallar puntos de partida naturales ajenos a las tradiciones culturales», sino «etnocéntricamente» de «limitarse a comparar y contrastar tradiciones culturales» (CP, xxxvii). ${ }^{13}$ El presupuesto de esta limitación, efecto de sustituir la Filosofía por la filosofía, es que «la filosofía no tiene una esencia propia en mayor medida que la literatura o la política. Cada una es lo que luego algunos hombres brillantes han hecho de ella. [...] Pero, aunque la filosofía no tiene esencia, sí tiene historia» $(\mathrm{CP}, 62)$. He ahí la paradoja, la retorsión implícita de la tradición, hay que superar la tradición filosófica no por ser tradición, sino porque la tradición nunca ha aceptado que la filosofía sólo sea una tradición (literaria): $:{ }^{14}$ «Uno no puede siquiera buscar una esencia de la filosofía en cuanto

13 La comparación se hace extensiva a las tradiciones antiteóricas y a las orientales en EHO, 71-3. Y es que «[1]a cuestión realmente en disputa es la del lugar de la Filosofía dentro de la filosofía occidental» (CP, xxix). De hecho, «[1]a autoimagen de un filósofo [...] depende casi enteramente de su concepción de la historia de la filosofía» (CP, 41). «El relato de la historia de la filosofía es una de las herramientas más poderosas de persuasión» (EOC, 24). Cf. «The Historiography of Philosophy» (TP, 247-73).

14 «La historia de esos conatos y de sus críticas es aproximadamente coextensiva a la historia del género literario que llamamos <filosofía〉, un género fundado por Platón. Así que los pragmatistas consideran que la tradición platónica ha perdido su utilidad» (CP, xiv [cf. EHO, 24]). 
Fach académico [...]. La filosofía se ve mejor como género de escritura. Está limitada, como cualquier género literario, no por la forma o la materia, sino por la tradición: una novela familiar que incluye, v. gr., al Padre Parménides, al viejo y honesto Tío Kant y al hermano malo Derrida» (CP, 92).

He aquí un «ambiguo», ciertamente paradójico, tradicionalismo. Así como Rorty se atrevió a usar el llamativo término «etnocentrismo» para designar otra cosa distinta de su significado habitual invirtiéndolo más bien, pues muestra, por así decir en abstracto, a los universales como particulares europeos sin fundamento, ${ }^{15}$ podemos aplicar cum grano salis la categoría de «tradicionalismo» (asimilable a su «historicismo») para su enfoque de la tradición, pues, bien mirado, quien siempre quiso escapar de la tradición fue el planteamiento tradicional, que de suyo buscaba salirse del tiempo y de la historia, así pues, de la tradición. Es propio de la Epistemología eternizar su tiempo, argumentar cómo los predecesores han errado en la conquista de la Verdad; frente a ella, el etnocentrismo sitúa a la hermenéutica, en la que ve «una expresión de la esperanza de que el espacio cultural dejado por la remisión de la epistemología no se llene» (PMN, 315) ${ }^{16}$ o sea, una «tradición» que asume su condición

15 El etnocentrismo no es incompatible con cierto universalismo (incluso, según el «Prólogo a la edición española» de AC, con una «federación planetaria» [cf. R. Rorty, Forjar nuestro país, tr. R. J. del Castillo. Barcelona: Paidós, 1999, p. 17]) siempre que éste se entienda como creado, por extensión de la consideración de quién es «uno de los nuestros», y no como dado naturalmente, presupuesto tradicional que «nos parece una manera engañosa de expresar la esperanza, que compartimos, de que la especie humana en su conjunto se convierta gradualmente en una comunidad global, en una comunidad que incorpore la mayor parte de la consistente moral de las democracias europeas industrializadas» (RFM, 47). De ahí este caveat: «la búsqueda de una comunidad humana universal conducirá a su propio fracaso si intenta preservar los elementos de cada tradición intelectual [...]. Se alcanzará, si acaso, mediante actos de creación, más que de descubrimiento, por un logro poético más que Filosófico» (CP, xxx). Luego se trata siempre de «rechazar la afirmación de la superioridad <objetiva de occidente, donde <objetiva〉 tiene un sentido ahistórico, transcultural» (TP, 199), persuadiendo «a nuestra sociedad de que la lealtad a ella misma es suficiente moralidad, y que semejante lealtad no precisa ya respaldo ahistórico» (ORT, 199).

16 «La hermenéutica es, más bien, lo que obtenemos cuando ya no somos epistemológicos» (PMN, 325). Pero repárese en que hermenéutica y Epistemología están en planos distintos. Sería erróneo entender que deberíamos ir linealmente de la Epistemología-sistemática a la hermenéutica-edificante. Ésta es parasitaria y reactiva: los filósofos edificantes «se mofan, satirizan y desdeñan -de diversos modos- la tradición. Son los maestros del discurso indirecto. Con semejantes técnicas se mofan del gran o/o» (Nielsen 1986b, p. 278). Se trata de proseguir la tradición retorciéndola, no proponiendo nuevos fundamentos y métodos alternativos a los tradicionales (se retuerce la idea misma -no sólo la vieja- de fundamento y método). Nielsen estudia la (meta)crítica rortiana a la tradición, y siendo él mismo crítico con Rorty (Nielsen 1986b, pp. 287-8) muestra cómo las defensas de la tradición más conocidas contra su «disolución» rortiana, que «está bien hecha» (Nielsen 1986b, p. 277), pese a su extrema plausibilidad, no «desvían de 
temporal y contingente de tradición, y que, en consecuencia, ha de declinarse en plural. ${ }^{17}$ Podríamos interpretar que mientras que para el planteamiento tradicional, en cuanto epistemológico, la tradición es investigación temporal conducente a lo intemporal, al texto sin contexto, matriz neutra, universal e inmutable, aspirando por ello a la cancelación de la propia tradición, para el «tradicionalismo» rortiano, en cuanto hermenéutico, la investigación temporal es «tradición» conducente siempre a lo temporal, al texto con-texto, matriz impura, particular y mutable, aspirando por ello a la proliferación de la tradición. ${ }^{18}$ Por eso, Rorty, antes que proponerse refutarla, quiso circunvalar la tradición. ${ }^{19}$

la arremetida subyacente en la crítica de Rorty a lo que ha venido a ser la tradición en filosofía» (Nielsen, 1986a, p. 19 [ $c f$. Nielsen 1991, 1999]).

17 Además de la ambigüedad «real», que permite pretender superar la tradición platónicokantiana y a la vez decir que «esas palabras -las de Platón y las de Kant- ciertamente han contribuido a que algunos de nosotros [...] seamos lo que somos» (TP, 350), hay otra ambigüedad «nominal», pues Rorty al hablar de la «tradición filosófica» está tomando la (mayor) parte por el todo, reduciendo metonímicamente la tradición a la platónico-kantiana, la de los «filósofos sistemáticos», cuando es plural: hay otras tradiciones. De hecho, frecuentemente Rorty se adscribe, por ejemplo, a la «nominalista», la «postnietzscheana», la «pragmatista americana», la «continental», etc., tradiciones que pueden identificarse como las de los «filósofos edificantes», aunque por su propia idiosincrasia no siempre formen una «tradición», pero casi (cf. PMN, 367). Buena parte de la ambigüedad nominal se podría haber atenuado si el objeto directo del verbos «superar» no hubiese sido la «tradición filosófica», sino mejor la «filosofía tradicional» (cuyo retrato se encuentra ya en PMN, 3) o, mejor aún, si Rorty hubiese restringido, como hace alguna vez, el alcance de la superación a «una determinada concepción tradicional de la filosofía» (ORT, 75 [cf. TP, 317]). Y es que, cuando falta la posibilidad de referencia a la «naturaleza», lo que queda es la historia, hay que arreglárselas con la «tradición». En cualquier caso, la concepción rechazada es la obediente a lo que Nietzsche llamó la «voluntad de sistema», que presupone poder poner punto final al proceso de creación escapando así de nuestra contingencia, de nuestras tradiciones (cf. CP, xix; Kolenda 1990, p. 113).

18 Así como «ningún lenguaje se ajusta más a la realidad que cualquier otro» (TPP, 22), no hay tradición más ajustada a la realidad que otra. Esta tesis va asociada a la de que la búsqueda de la objetividad lo es de un acuerdo entre sujetos, algo que requiere de mucha retórica. Y esto nos permite interpretar el «amor a la verdad» antes como «amor» a los otros que a lo otro ( $c f$. TPP, 25), es decir, ligar la racionalidad, en lugar de a la objetividad, a la intersubjetividad o a la lealtad recíproca o a la solidaridad ( $c f$. ORT, 13,45$)$ o a la esperanza $(c f$. PSH) o al «quietismo» (cf. PCP, 147-59).

19 Ejemplo conspicuo de rodear la tradición, en lugar de atravesarla, son el primer ensayo sobre Derrida, «Deconstruction and Circumvention» (EHO, 85-106), y este comentario: «no es la huida de la tradición filosófica lo que Derrida tiene en mente. Nos previene contra la idea de que el «discurso filosófico〉 sea «meramente metáforas olvidadas o gastadas, una fábula particularmente gris y triste, mistificada hasta el punto de proponerse a sí misma como la auténtica verdad» (TP, 332), al que añade en nota: «Con algunas matizaciones (como la de sustituir 〈filosófico〉 por «metafísico〉), así es exactamente como yo efectivamente pienso el discurso de la tradición filosófica». Años atrás ya había advertido que «el pragmatista no intenta justificar 
Más que de un tomar un atajo se trata de dar un rodeo para disuadirla de sus tendencias «suicidas» y hacerla «sostenible».

Rorty no tenía ni podía tener argumentos fuertes (no-circulares) contra la tradición, ${ }^{20}$ pues eso habría sido tomar de ella justo lo que repelía; si acaso ofrecía consejos para cambiar, mejorándolas, mediante la «persuasión racional», ${ }^{21}$ nuestras vidas «europeas». Y esos consejos sólo se pueden dar practicando la conversación tradicional (en el doble sentido del genitivo implícito), precisamente desviándose de los constantes «esfuerzos de la tradición filosófica por escapar del tiempo y la historia» (CP, 174), para ejercer la virtud socrática, virtud no epistémica sino moral, ${ }^{22}$ siendo leales a Sócrates donde Platón y su tradición no lo fueron. Estamos, esperanzas de la tradición mediante, ante «la idea de la cultura como conversación más que como estructura erigida sobre fundamentos» (PMN, 319), de la «conversación como el contexto último dentro de cual se debe entender el conocimiento» (PMN, 389) y del filósofo como «el diletante informado, el polipragmático intermediario socrático entre varios discursos» $(\mathrm{PMN}, 317) .{ }^{23} \mathrm{Y}$ el interés de esta filosofía etnocéntrica («Sólo hay diálogo», sólo nosotros» [ORT, 32]) «es seguir manteniendo la conversación más que encontrar la verdad objetiva» (PMN, 377), una conversación que podríamos considerar «autotélica», como su propio fin, un fin sin final. ${ }^{24}$ Este

sus metáforas mediante un argumento filosófico [...], cuenta historias sobre cómo las metáforas que detesta han puesto en ridículo el curso del pensamiento occidental» (ORT, 82). Sobre el «repudio de la tradicional imagen logocéntrica» véase EHO, 132 ss.

20 Los filósofos edificantes «rechazan la epistemología y la metafísica en cuanto disciplinas posibles. Hablo de 〈dar de lado〉, en vez de 〈argumentar en contra〉, porque su actitud hacia la problemática tradicional es como la de los filósofos del siglo XVII hacia la problemática escolástica» (PMN, 6 [cf. PMN, 365-72]).

21 Para Rorty «〈racional〉 significa meramente 〈persuasivo»» (ORT, 220): «Me gustaría que separáramos la noción de racionalidad de la de verdad. Quisiera definir la racionalidad como el hábito de lograr nuestros fines mediante la persuasión y no por la fuerza. [...] [L]a oposición entre racionalidad e irracionalidad no es ni más ni menos que la oposición entre palabras y golpes» (EOC, 28). Sobre el motto «persuasion rather tan force» véase, v. gr., ORT, 39, 67, 218). Y es que filosofía y retórica se entreveran: «La persuasión es tan frecuentemente una cuestión de hacer que la gente deseche un vocabulario (y las cuestiones que ella enuncia en su seno) como de argumentación deductiva» (PP, 463).

22 Sobre la inexistencia de una virtud llamada «racionalidad» distinta de las virtudes «morales» véase ORT, 61, y repárese en que «[m]i rechazo de las nociones tradicionales de la racionalidad puede resumirse diciendo que el único sentido en que la ciencia es ejemplar es que es un modelo de solidaridad humana» (ORT, 39).

23 Frente al modelo socrático-edificante de la «tertulia» (entre generaciones, culturas y tradiciones [cf. PF, 203]), tenemos el platónico-sistemático tradicional de la «supervisión cultural»: «El primer papel es el adecuado para la hermenéutica, el segundo para la epistemología» (PMN, 318).

24 Esta conversación carece de toda garantía metafísica o epistemológica, nada fundamenta su éxito, salvo su continuidad. Así que pecar contra Sócrates sería siempre en todo caso 
interés, al que, entendemos, subyace la solidaridad ${ }^{25}$ con el pasado, es moral: «El único punto en que yo insistiría es que el interés moral del filósofo ha de ser continuar la conversación de Occidente, más que exigir un lugar, dentro de esa conversación, para los problemas tradicionales de la filosofía» (PMN, 394 [cf. CP, 172]). Y es que no hay en Rorty una argumentación alternativa a la de la tradición, sino más bien una alternativa ${ }^{26}$ a la argumentación de la tradición, un cambio de retórica, ${ }^{27}$ cuya «ambigua ${ }^{28}$ moraleja es: «Mantente en contacto

pecar contra nuestra comunidad, nunca contra la naturaleza: «no hay ninguna plataforma de observación supercultural desde la que situarnos» (ORT, 213).

25 Aunque Rorty no lo plantee así, al igual que la tradición es el fondo sobre el que ejercer parasitariamente la ironía, también es un fondo solidario, en tanto que atesora las esperanzas de nuestros ancestros, y que sólo en ininterrumpida conversación con él podremos expresar las nuestras. Su comentario a un poema de Larkin (CIS, 23-6, 40) es ejemplo de cómo la relación con nuestro pasado aparece bajo otra luz cuando consideramos qué actitudes nos gustaría que asumiesen nuestros descendientes respecto de nuestros valores. La glosa deja ver que el poeta contempla sus obras siempre como inacabadas, incapaces de agotar todo lo que él quisiera expresar. La posibilidad de su compleción depende de los extraños y futuros lectores y creadores, de su actitud ante el legado por ellos heredado, ante la tradición. Lo que Rorty dice del poeta podría decirse también de su propia relación con la tradición, como una muestra de los usos del pasado ( $c f$. Kolenda 1990, pp. 43-6).

26 «L que es erróneo en esta cuestión es que, como era preguntada por Sócrates y la tradición platónica, presupone que nosotros sabemos qué es el conocimiento [...]. El modo correcto de plantear esa cuestión es «¿Por qué encuentras persuasivo lo que acabas de decir?». Ésta es una cuestión que hace caso omiso de las tradicionales distinciones entre razones y causas, psicología y lógica, retórica y demostración» (PP, 463).

27 «Nuestros esfuerzos de persuasión deben adoptar la forma de una gradual inculcación de una nueva manera de hablar y no de una argumentación directa en el seno de viejas maneras de hablar» (RFM, 34). Se trata no de cambiar filosofía por retórica, sino -«abandono de la ficción heurística de un público universal» mediante- de «cambiar de retórica», pues «nunca vamos a encontrar un argumento irresistible e invariante respecto del auditorio [...], un argumento que sea puramente 〈lógico〉 y en absoluto 〈retórico»» (TP, 284-5). Respecto de este cambio de práctica retórica, en particular, podemos decir lo que Rorty afirma respecto de su cambio de prácticas, en general, que es «tan indemostrable y tan intuitivo como lo era la tradición racionalista occidental» (TP, 83). Por eso es «retórico» y no «lógico», como lo era la tradición sin saberlo y, por supuesto, sin quererlo. Por cierto, en el abandono del auditorio universal, inherente al «etnocentrismo», encontramos la regla de oro de la retórica, la adaptación del discurso al auditorio: «Los argumentos sólo funcionan si el vocabulario en que establecer premisas es compartido por hablante y auditorio» (EHO, 93 [cf. EHO, 134]). Que «la justificación siempre es relativa a un auditorio» (TP, 4, 22 [cf. ORT, 126-50]) hace sospechosa la distinción entre verdad y justificación ( $c f$. PSH, 36-8) o entre validez genuina y manipulación retórica, pues la diferencia entre una y otra se reduce a la diferencia entre viejos y nuevos auditorios (lo que sería extrapolable a las distintas tradiciones).

28 De Rorty acerca de la tradición acaso se pueda decir lo que él de Derrida: «uno puede preguntarse si la única forma de no ser un griego es ser un judío, y si la única escapatoria a la metafísica de la presencia es convertirse, como dice Derrida, en judeogriego y en griegojudío» (TP, 342). 
con ella justo lo suficiente para recordarte a ti mismo que sería inhumano ignorarla y fatal tomársela demasiado en serio. Recuerda que desde un punto de vista pragmático, no siempre compensa ser un pragmatista» $(\mathrm{TP}, 341) .{ }^{29}$

\section{IV}

Esta «ambigüedad» de la relación con la tradición se extiende particularmente a la cuestión del «final de la filosofía». Por ello, más que por especial énfasis rortiano al respecto, es frecuente encajar a Rorty dentro de los pensadores que han proclamado la «muerte de la filosofía». ${ }^{30}$ De hecho, sería tarea fácil aislar frases suyas para avalar semejante interpretación. El propio Rorty fue consciente de que su propuesta de superar la tradición filosófica se puede asimilar a ese tópico actual y tanto lo desmintió rotundamente, por un lado, como, por otro, intentó ofrecer una explicación de esa asimilación. En cualquier caso, quede claro como conclusión anticipada que «abandonar a Platón y Kant no es lo mismo que abandonar la filosofía» (PF, 198). Es decir, el propósito de Rorty, a lo sumo, es el de cambiar la dirección de un ramal de la conversación filosófica, no liberarnos de la ilusión filosófica, ni llevar la filosofía a un final. ${ }^{31}$

29 Hay que «atender siempre la voz del pasado» y simultáneamente «persuadir a la gente a veces y en casos excepcionales de hacer oídos sordos a esa voz» (TP, 137).

30 Cf. Putnam 1990, Bernstein 1991, Habermas 1996, Haack 1998. No entramos en la discusión de cada uno de estos planteamientos, en particular, ni de los que, en general, juzgan la precisión de Rorty al tratar la tradición (calculada en broma como «factor Rorty» en 0,742 por Dennet 1982, p. 349), porque lo que queremos destacar es que el problema del «fin de la filosofía» sólo se plantea como tal problema desde un marco que es el que Rorty, con todo, desplaza al (más que argumentar contra la tradición) cambiar de retórica. Como dicen Guignon \& Hiley 2003, p. 33: «Lo que es sorprendente y a menudo fastidioso para los filósofos que intentan formular semejantes objeciones es la tendencia de Rorty a evadirlos socavando la entera línea de argumentación» [lo que valdría para Taylor 1990]. Con independencia de con quien se esté de acuerdo, el desacuerdo es más «sintáctico» que «semántico», estriba en partis pris distintos, en su diversa retórica (o por decirlo con resonancias davidsonianas, en el «esquema» antes que en el «contenido»; o por decirlo con resonancias heideggerianas, en una «transformación del pensar, no en un enunciado sobre un contenido»). Por eso, no podemos «verter vino nuevo en botellas viejas, y escribir de una forma continua con la tradición filosófica» (ORT, 161).

31 Esta tesis la encontramos también en Kraugerud \& Ramberg 2010. Añadamos que el cambio de dirección no obedece a la falsedad de la tradición circunvalada, sino a su ineficiencia. Y es que apreciamos que la noción operativa en Rorty de verdad está más cerca de una versión secularizada del emet de la tradición religiosa judeo-cristiana que de la alétheia de la tradición «objetivista», esto es, de una verdad que no se descubre, sino que se hace ( $c f$. Jn 3, 21; I Jn 1,6), y que, por consiguiente, no se opone al error o a la falsedad, sino a la ignavia (a la «infidelidad» a Dios o, en el caso rortiano, al «nosotros» etnocéntrico). La verdad rortiana, como la religiosa, no se conoce, sino que se practica, se hace en el tiempo, se hace tradición. He ahí la primacía 
El mentís expreso distingue entre «desear el fin del plan de estudios de filosofía» y «desear el fin de la filosofía». Rorty sí quiere lo primero, siempre que tengamos en cuenta que se refiere contextualmente al plan de estudios de los departamentos de filosofía dominados por la tradición analítica, pero no lo segundo: «Quizá pueda aclararse un poco el asunto si digo que espero que la gente no deje nunca de leer, v. gr., a Platón, Aristóteles, Kant y Hegel, pero que asimismo espero que, tarde o temprano, se deje de engatusar a los novatos para que se interesen por el problema del mundo externo y por el problema de las otras mentes» (TP, 47, n. 16). Y se remonta a su veterano «rechazo explícito», cuando reconocía que si bien su rechazo de la paradigmática imagen especular puede dar lugar a que se entienda que no podría o no debería darse la profesión de filósofo: «eso no se sigue. Las profesiones pueden sobrevivir a los paradigmas que les dieron nacimiento. En cualquier caso, la necesidad de profesores que hayan leído a los grandes filósofos muertos es completamente suficiente para asegurar que siga habiendo departamentos de filosofía mientras siga habiendo universidades [...]. Pero ocurra lo que ocurra, no hay peligro de que la filosofía 〈llegue a su fin»» (PMN, 393-4). ${ }^{32}$

Si ya fue tan explícito en los setenta, podemos preguntarnos por qué décadas después sigue atribuyéndosele la recomendación del fin de la filosofía. Él mismo intenta razonarlo ya a finales de siglo así: «Mi primer impulso, cuando me cuentan un enigma filosófico, es intentar disolverlo en vez de resolverlo. [...] Esta clase de conducta puede dar cuenta del hecho de que a menudo se me caracterice como un filósofo del final-de-la-filosofía, pero no lo soy. La filosofía no puede llegar a su final mientras el cambio cultural no llegue a su final y, como cualquiera, espero que semejante cambio continúe. Dado el cambio cultural, siempre habrá gente que intentará unir lo viejo con lo nuevo» (APTP). Luego es el proceder de Rorty, su ironista «método» de la «redescripción», de tan wittgensteiniano eco, la razón de que, según confiesa, se le adjudique la etiqueta de vocero del «fin de la filosofía».

Y seguramente sea en un ensayo sobre Wittgenstein, «Keeping Philosophy Pure» (CP, 19-36), donde se encuentren las claves de esta razón, toda vez que se manifiesta a plena luz lo que hay aquí en juego, que no es otra cosa que la concepción de la filosofía misma. Ahí Rorty se pregunta «Tiene sentido decir que una nueva concepción filosófica comporta la muerte de la filosofía? En particular, ¿tiene sentido decir que la filosofía de algún modo ha sido superada, ha

pragmatista del hacer sobre el contemplar, palmaria en la preferencia rortiana por la metáfora de coping, en lugar de la de copying, a la hora de invitarnos a imaginar la misión del conocimiento, en particular, y de la filosofía, en general, toda vez que de la dimensión intelectual de la noción de coping son inescindibles dos dimensiones más: acción y esperanza ( $c f$. Kolenda 1990, pp. 22-6).

32 La necesidad de «aproximación a los grandes filósofos» $(\mathrm{CP}, 68)$ salvaguarda a la filosofía de su fin. 
dejado de estar de moda o se ha marchitado a resultas de cierto descubrimiento que hizo Wittgenstein [...]?» (CP, 22). Aquí empieza a oler a la inevitable muerte de la filosofía, y Rorty hace palmaria la cuestión del «fin de la filosofía». Y la plantea mediante una serie de dilemas, en que ahora no podemos entrar, aunque destaquemos que en el cuerno de uno de ellos Wittgenstein «está jugando con nuestros sentimientos mezclados acerca de la tradición filosófica» (CP, 27). Para escapar a esos dilemas Rorty distingue tres acepciones de la palabra «filosofía», dos de ellas no se verán afectadas por el final, pero la restante sí.

En efecto, como «especialidad académica» no es cuestión su final ni el de la «pureza» de sus problemas tradicionales, dados contextualmente por un determinado corpus bibliográfico definitorio: «Esta pureza no es exclusivamente "filosófica"; también la poseen el estudio de los fluoruros y el de la prosodia de Chaucer. En este sentido, tampoco se da el problema de ponerle o no fin a la filosofía» (CP, 30-1). Tampoco se suscita en el caso de la «filosofía visionaria», que es como Rorty llama a la filosofía como género de escritura generalísimo dedicado a la comprensión de la interrelación de las cosas, pues en este «sentido, la filosofía obviamente no es un Fach de por sí, y nadie clamaría nunca por su <pureza〉. Ni nadie tampoco pensaría que se pueda o deba ponerle fin» $(\mathrm{CP}, 30)$.

Sin embargo, hay una tercera acepción, la de «la filosofía como nombre dado al estudio de ciertos problemas definidos y permanentes»- (CP, 31), los de la «tradición cartesiana», que Rorty hace extensiva a la «entera tradición cultural que hizo de la verdad [...] una virtud central» (CP, 35), anejos a la noción tradicional de «necesidad» ${ }^{33}$, a los que las Investigaciones nos permiten dar carpetazo. El final de la filosofía se refiere únicamente a esta concepción: «afirmar que las Investigaciones podrían poner fin a la filosofía sólo puede significar que este libro podría de algún modo desembarazarnos de la <imagen que nos tiene cautivos), de la imagen del hombre que genera los problemas tradicionales. Afirmar que la filosofía podría finalizar no equivale a afirmar que tener concepciones amplias pueda dejar de estar de moda, o que los departamentos de filosofía puedan ser enterrados, sino más bien que determinada tradición cultural puede desaparecer» (CP, 32). De todas maneras, ese final tampoco es inminente: «¿Pueden unos cuantos volúmenes satíricos tirar por tierra una tradición de trescientos años? Ciertamente no. [...] El fin de la filosofía-como-sucesora-de-la-teología, de una disciplina 〈pura〉 en la que se aborden problemas profundos mediante métodos apropiadamente puros, no llegará en nuestro tiempo» $(\mathrm{CP}, 34)$.

33 Rorty confronta su sentido «literario» de la contingencia con la necesidad «literal» de la tradición onto-teológica, para la que lo contingente (como lo metafórico) se asocia con lo aparente ( $c f$. EHO, 34), lo que apuntaría nuevamente a la «centralidad» retórica de su planteamiento. 
Y es que para Rorty la filosofía siempre termina por enterrar a los que pronuncian su discurso funerario, porque los tiempos no paran de cambiar ( $c f$. APTP). ${ }^{34}$ Lo único que él esperaba al hablar del «fin de la filosofía» era casi lo mismo que Heidegger: ${ }^{35}$ que terminasen «los intentos de llevar a la filosofía por el camino seguro de una ciencia» (APTP). Y nada más. De lo contrario, la cuestión degenera en un pseudotema, tan banal como «el final de la novela», y su anuncio no puede menos que ser saludado con «cinismo». ${ }^{36}$ Así que a cualquier pretensión de redactar apelando a la autoridad de Rorty el obituario de la tradición filosófica tout court habría que darle la misma respuesta que Mark Twain telegrafió al periódico que notificó su propia defunción: «noticias sobre mi muerte demasiado exageradas».

\section{CONCLUSIONES}

La relación de Rorty con la tradición filosófica presenta más matices de los que su reputación hacía creer. Aunque se ha ganado una bien merecida fama como pensador que quiere dejar atrás el pasado filosófico en aras de una cultura postFilosófica y buena parte de su pensamiento (de hecho, las bases del mismo) denota un contundente ironismo frente a la tradición, no es menos básica, pese a que la crítica no haya apenas reparado en ello, su solidaridad con el legado filosófico en tanto que es depósito de las esperanzas de nuestros ancestros, y que sólo en ininterrumpida conversación (por parasitaria que sea) con él podremos expresar las nuestras. Así pues, su posición frente a la tradición

$34 \ll[C]$ ada libro-sobre-el-fin-de-la-filosofía parece a menudo justo otro libro de filosofía más» (TP, 343). Y es que el «historicismo» rortiano sabe que circunvalar el logos tradicional comporta automutilarnos, privarnos de lo que nos ha hecho ser lo que somos.

35 Salvo matices, Rorty adhiere al planteamiento heideggeriano del Ende der Philosophie y «[s]us comentarios sobre la tradición» (CP, 39). Diéguez 2003 estudia la cuestión rortiana del final de la filosofía relacionándola con su tratamiento heideggeriano, bajo la hipótesis de que su doble lectura, radical (de toda filosofía) o moderada (de un tipo determinado), está a la base de los malentendidos propiciados por el propio Rorty. Más tajante es Kalpokas 1999 y 2005: el rechazo rortiano del valor cognitivo-argumentativo de la filosofía equivaldría sin más a su final.

36 Parafraseando un curioso pasaje donde Rorty trata del fin de la filosofía como algo de otros, no suyo: «Hablar del 〈final de la filosofía〉 resulta tan fácil, pero tan huero, como hablar del «final de la novela〉. [...T] odos saben que «la muerte de la novela〉 no significa nada más que 〈a muerte de un determinado tipo de novela〉. Deberíamos sentir un parecido cinismo acerca de los anuncios del 〈fin de la filosofía〉, que típicamente significan algo así como <el fin de la construcción de sistemas metafísicos〉, o <el fin del empirismo〉, o <el fin del cartesianismo〉. Ninguna definición de 〈filosofía〉 puede cubrir a Carnap y a Rawls, al primer Hegel y al último Wittgenstein, a Derrida y a Habermas, y aún así aislar algo lo suficientemente coherente como para tener un 〈fin〉» (TP, 317). 
no es simplemente de unilateral rechazo, sino más bien ambigua. Sin embargo, la ambigüedad queda a salvo de la inconsistencia en la medida en que Rorty no pretendió tanto ofrecer una argumentación alternativa a la de la tradición (atajarla) cuanto una alternativa a la argumentación de la tradición (rodearla). Por tanto, la aportación original del presente trabajo estriba precipuamente en, aparte de enfatizar el hecho antes no del todo percibido como tal de la complejidad de la posición rortiana ante la tradición filosófica, apuntar como novedosa clave interpretativa del mismo un cambio de retórica, lo que secundariamente permite la posibilidad, a su vez, de un enfoque distinto al habitual para el abordaje de la cuestión del «final de la Filosofía».

\section{REFERENCIAS BIBLIOGRÁFICAS}

I. Fuentes citadas de R. Rorty:

[LT] (ed.), The Linguistic Turn: Recent Essays in Philosophical Method. Chicago: UCP, 1967.

[PMN] Philosophy and the Mirror of Nature. Princeton: PUP, 1979.

[CP] Consequences of Pragmatism. Minneapolis: UMP, 1982.

[PP] «Philosophy without Principles», Critical Inquiry, 11/3 (1985), pp. 459-65.

[CIS] Contingency, Irony, and Solidarity. Cambridge: CUP, 1989.

[ORT] Objectivity, Relativism, and Truth: Philosophical Papers, Volume 1. Cambridge: CUP, 1991.

[EHO] Essays on Heidegger and Others: Philosophical Papers, Volume 2. Cambridge: CUP, 1991.

$[\mathrm{PF}]$ «Philosophy and the Future», en H. J. Saatkamp (ed.), Rortyand Pragmatism. Nashville: VUP, 1995, pp. 197-206.

[EOC] «Emancipating Our Culture», en J. Niznik \& J. T. Sanders (eds.), Debating the Estate of Philosophy: Habermas, Rorty, and Kolakowski. Westport: Praeger, 1996, pp. 24-30.

[RFM] «Relativism: Finding and Making», en J. Niznik \& J. T. Sanders (eds.), Debating the Estate of Philosophy: Habermas, Rorty, and Kolakowski. Westport: Praeger, 1996, pp. 31-47.

[TPP] Truth, Politics and 〈Post-Modernism〉. The 1997 Spinoza Lectures. Amsterdam: Van Gorcum, 1997.

[TP] Truth and Progress: Philosophical Papers, Volume 3. Cambridge: CUP, 1998.

[AC]Achieving Our Country: Leftist Thought in Twentieth Century America. Cambridge: HAP, 1998.

[JLL] «Justice as a Larger Loyalty», en Ph. Cheah \& B. Robbins (eds.), Cosmopolitics: Thinking and Feeling beyond the Nation. Minneapolis: UMP, 1998, pp. 45-58.

[PSH] Philosophy and Social Hope. New York: Penguin, 1999. 
[APTP] «Analytic Philosophy and Transformative Philosophy». Disponible sólo en línea: <http://www.evans-experientialism.freewebspace.com/rorty02.htm> [Consulta: 30-11-09].

[PGT] «Persuasion is a Good Thing», en E. Mendieta (ed.), Take Care of Freedom, and Truth will Take Care of itself: Interviews with Richard Rorty. Stanford: SUP, 2006, pp. 66-88.

[PCP] Philosophy as Cultural Politics: Philosophical Papers, Volume 4. Cambridge: CUP, 2007.

II. Otras referencias:

ABRAMS, J. J. 2002: «Philosophy after the Mirror of Nature: Rorty, Dewey, and Peirce on Pragmatism and Metaphor», Metaphor and Symbol, 17/3, pp. 227-42.

BERNSTEIN, R. 1991: The New Constellation. Cambridge: Polity Press.

CALDER, G. 2003: Rorty: and Redescription. London: Weidenfeld \& Nicolson.

DENNETT, D. 1982: «Comments on Rorty», Synthese, 53, pp. 349-56.

DIÉGUEZ, A. 2003: «La necesidad de la epistemología en un mundo tecnificado. ¿Es Rorty inconsistente?», Éndoxa, 17, pp. 155-82.

GUIGNON, C. \& HILEY, D. R. (eds.) 2003: Richard Rorty. Cambridge: CUP.

HAACK, S. 1998: Manifesto of a Passionate Moderate, Chicago: UCP.

HABERMAS, J. 1996: «Coping with Contingencies. The Return of Historicism», en J. Niznik \& J. T. Sanders (eds.), Debating the Estate of Philosophy: Habermas, Rorty, and Kolakowski. Westport: Praeger, 1996, pp. 24-30.

KALPOKAS, D. 1999: «¿Superación de la epistemología o final de la filosofía?», Revista de Filosofía, XI/22, pp. 255-85.

- 2005: Richard Rorty y la superación pragmatista de la epistemología. Bs. Aires: Ediciones del Signo.

KOLENDA, K. 1990: Rorty's Humanistic Pragmatism: Philosophy Democratized. Gainesville, FL: University of Florida Press.

KRAUGERUD, H.-A. \& RAMBERG, B.-T. 2010: «The New Loud: Richard Rorty, Quietist?», Common Knowledge 16/1, pp. 48-65.

NIELSEN, K. 1986a: «Rorty and the Self-image of Philosophy», International Studies in Philosophy, XVIII/1, pp. 19-28.

- 1986b: «Scientism, Pragmatism, and the Fate of Philosophy», Inquiry, 29, pp. 277-304.

- 1991: «The Tradition in Retreat», en After the Demise of Tradition: Rorty, Critical Theory, and the Fate of Philosophy. Boulder, CO: Westview Press, pp. 39-55.

- 1999: «Taking Rorty Seriously», Dialogue, XXXVIII, pp. 503-18.

OCAMPO, A. 2004: «Reflexiones en torno a la propuesta ética de la sentimentalidad» [en línea], Razón y Palabra, 40 <http://www.razonypalabra.org.mx > [consulta: 10-09-09].

PUTNAM, H. 1990: Realism with a Human Face, Cambridge: HUP.

TAYLOR, C. 1990: «Rorty in the Epistemological Tradition», en A. Malachowski (ed.), Reading Rorty. Oxford: Blackwell, pp. 256-75. 
José A. Marín-Casanova es profesor en el Departamento de Metafísica y Corrientes Actuales de la Filosofía, Ética y Filosofía Política de la Universidad de Sevilla

Publicaciones recientes:

2007: El pensamiento en forma. De metáfora, retórica y democracia. Sevilla-Madrid: Ediciones Parthenon.

2009: Contra natura. El desafío axiológico de las nuevas tecnologías. Sevilla: Ediciones Paso-Parga.

Líneas de investigación:

Pensamiento contemporáneo, retórica filosófica, filosofía de la tecnología

Dirección postal:

C/ Camilo José Cela, s/n

41018 Sevilla

Dirección electrónica:

jamarin@us.es 